Review began 01/20/2022 Review ended 01/29/2022 Published 01/31/2022

\section{(๑) Copyright 2022}

Mishra et al. This is an open access article distributed under the terms of the Creative Commons Attribution License CC-BY 4.0. which permits unrestricted use, distribution, and reproduction in any medium, provided the original author and source are credited.

\title{
Study on the Effect of Berberine, Myoinositol, and Metformin in Women with Polycystic Ovary Syndrome: A Prospective Randomised Study
}

\author{
Neha Mishra ${ }^{1}$, Ruchi Verma ${ }^{1}$, Payal Jadaun ${ }^{2}$ \\ 1. Obstetrics and Gynaecology, Goverment Institute of Medical Sciences, Greater Noida, IND 2. Obstetrics and \\ Gynaecology, Veerangana Avantibai Lodhi Autonomous State Medical College, Etah, IND
}

Corresponding author: Neha Mishra, imneha2908@gmail.com

\begin{abstract}
Objective: This study was conducted to see the effects of berberine, metformin, and myoinositol in women with polycystic ovary syndrome (PCOS).

Materials and Methods: Subjects were randomly assigned via computer-generated randomization to one of the three treatment groups. Group 1 received berberine hydrochloride $500 \mathrm{mg}$ twice daily, group 2 received metformin hydrochloride $500 \mathrm{mg}$ twice daily, and group 3 received myoinositol $1000 \mathrm{mg}$ twice daily to compare clinically (mean weight, waist circumference, waist-to-hip ratio, and body mass index), metabolic (fasting blood sugar, serum fasting insulin, fasting blood sugar/serum fasting insulin [FBS/FI]), hormonal effects (serum total testosterone [TT], serum sex hormone-binding globulin [SHBG] and free androgen index) together with the lipid profile (total cholesterol, serum triglyceride, serum low-density lipoprotein [LDL], very-low-density lipoprotein [VLDL], serum high-density lipoprotein [HDL]) in patients receiving metformin, berberine, and myoinositol before and after three months of treatment.
\end{abstract}

Results: Weight, BMI, waist circumference, waist-hip ratio, FBS, FI and fasting glucose/insulin ratio, total testosterone, free androgen index (FAI), SHBG, total cholesterol, triglycerides, LDL, VLDL, and HDL showed significant differences in three groups after three months of treatment $(p<0.0001)$. Between the three groups, berberine showed greater differences in clinical, hormonal, and lipid parameters compared to metformin and myoinositol, while myoinositol showed greater improvement in carbohydrate metabolic parameters.

Conclusions: Metformin, the classical drug used in PCOS, improves all the parameters in polycystic ovary syndrome women. Berberine may have greater potential to reduce the risk of cardiovascular disease than metformin in PCOS patients due to its effect on body composition, lipid profile, and improvement in hormone status. Myoinositol administration improves endocrine parameters and insulin sensitivity. It may be considered as a first-line option in PCOS patients with insulin resistance without prediabetes or diabetes.

Categories: Endocrinology/Diabetes/Metabolism, Family/General Practice, Obstetrics/Gynecology Keywords: polycystic ovary syndrome (pcos), serum fasting insulin, sex hormone binding globulin, free androgen index, waist circumference, waist hip ratio, metformin, myo-inositol, berberine

\section{Introduction}

Polycystic ovary syndrome (PCOS) is a heterogeneous endocrinopathy characterized by irregular menstruation, infertility, hyperandrogenemia, acanthosis nigricans, and a biochemical profile showing increased luteinizing hormone/follicle-stimulating hormone (LH/FSH) ratio, increased androgen levels, hyperinsulinemia, dyslipidemia, and obesity. In India, approximately $21 \%$ of reproductive-age women suffer from PCOS [1]. The burden of PCOS as a disease is immense. The short- and long-term sequelae resulting from PCOS call for shifting our focus to early diagnosis and timely treatment [2]. Its association with the development of diabetes, metabolic syndrome, cardiovascular disease, obstructive sleep apnea, endometrial carcinoma, depression, and anxiety has been known for a long time. Moreover, the combination of various phenotypes makes its management confusing and complex. Therefore, there is always a tendency among clinicians to opt for newer drugs for PCOS [3]. Metformin is one of the oldest drugs used to treat PCOS. Metformin is an oral antidiabetic drug of the biguanide class. The main effect of this drug is to decrease hepatic glucose production through a mild inhibition of the mitochondrial respiratory chain complex. Documentation of insulin resistance is good prior to recommending its use [4]. Berberine is a type of isoquinoline derivative alkaloid herb extract. It improves insulin sensitivity and reduces hyperandrogenaemia. Its main effects are increased insulin receptor expression, activation of AMP-activated protein kinase (AMPK), and stimulation of glucose uptake by GLUT-4 up-regulation. It has been administered to patients with diabetes mellitus. Recently, clinical studies have emphasized that AMPK activation is decreased in ovarian theca cells in PCOS women. This theory has caught the attention of researchers, and berberine is being explored regarding its utility in treating PCOS. Many studies have 
considered it a promising drug for treating PCOS in the future [4,5]. A new addition to the list of drugs used for PCOS is myoinositol. Myoinositol is a group of drugs that belong to the vitamin B family. It is a key factor in insulin signaling and also serves as a precursor to D-chiro-inositol in endogenous inositol metabolism. Myoinositol administration has a modulatory role in insulin sensitivity, gonadotrophin, and androgen secretion $[6,7]$.

Although metformin has shown widespread application in patients with PCOS, from maintaining body fat composition to facilitating successful ovulation index regimes, its poor tolerability profile, gastrointestinal side effects, and large tablet size affect patient compliance $[8,9]$. Berberine and myoinositol have shown promising effects in some studies [4-7]. Anthropometric characteristics, metabolic parameters, hormonal parameters, lipid profile, infertility, and future risk of metabolic syndrome: all these issues must be addressed in a timely manner in patients with PCOS. So, in the present study, we aimed to compare the effects of metformin, berberine, and myoinositol on the clinical, metabolic, endocrine, and lipid parameters of PCOS patients. This study also aimed to investigate whether the time-tested drug metformin in PCOS patients needs to be replaced by newer options. To the best of our knowledge, this is the first study to prospectively examine the effects of three drugs on patients with PCOS.

\section{Materials And Methods}

The present prospective study was done at Motilal Nehru Medical College, Allahabad over 24 months in PCOS women attending the outpatient department. Women with PCOS in the 15-40 age group were included in the study. The PCOS patients were defined as per Rotterdam criteria (any two of the following features: oligo-ovulation or an-ovulation, clinical or biochemical signs of hyperandrogenism or both, and polycystic ovaries as seen on ultrasound scanning [10]. Women diagnosed with systemic and endocrine disorders, lateonset congenital adrenal hyperplasia, Cushing's syndrome, thyroid disorders, hyperprolactinemia, and diabetes mellitus were excluded. Women taking medications known to alter insulin physiology, oral contraceptives, ovulation induction drugs, anti-obesity drugs, and undergoing in vitro fertilization treatment currently or in the last three months were also excluded from the study.

Taking the difference between the means under consideration as per previous studies, and keeping the power of the study at $80 \%$ with a $5 \%$ level of significance, the minimum sample size was calculated to be 129 . Taking into account the $5 \%$ loss to follow-up, the total sample size was 135 with 45 in each group [4,6].

A total of 230 women were screened in the outpatient department for the current study, and 136 were recruited after applying the inclusion and exclusion criteria. Details on recruitment are shown in Figure 1.

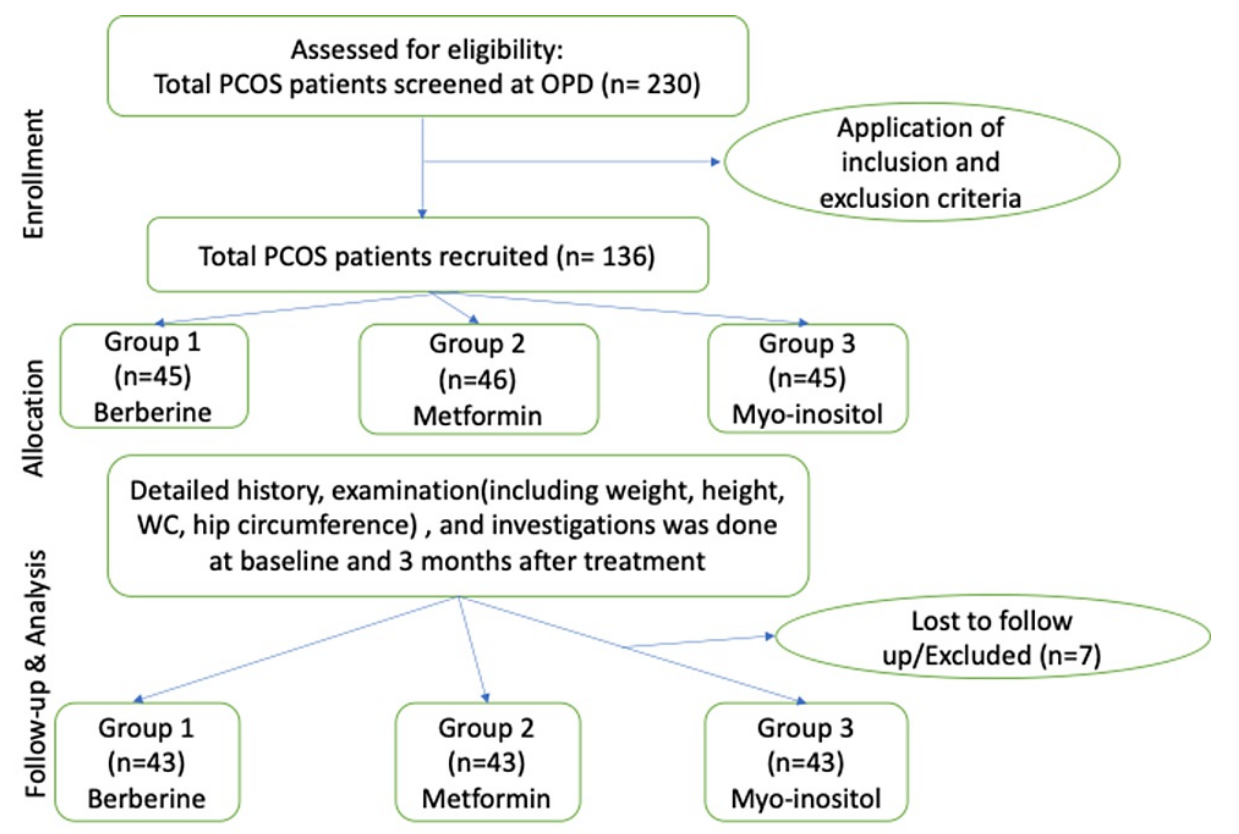

FIGURE 1: Recruitment flow chart

Approval was obtained from the ethics committee of the institution (IEC/MLNMC2014/No.02). Written informed consent was obtained from each participant. A detailed history was taken with special focus on a menstrual pattern such as infrequent menstrual periods (interval between menstrual periods $\geqslant 35$ days), amenorrhoea (absence of vaginal bleeding for at least 90 days), clinical hyperandrogenism (a FerrimanGallwey score $\geqslant 6$ ), and/or biochemical hyperandrogenism (total testosterone (TT) $\geqslant 58 \mathrm{ng} / \mathrm{dl}(2 \mathrm{nmol} / \mathrm{l})$ ) to 
A thorough clinical examination including general condition, built, height, weight, body mass index, waist/hip ratio, hirsutism characteristics (increased facial hair, body hair, acne), thyroid gland enlargement, acanthosis nigricans, any palpable lymph node, edema, pulse rate, blood pressure, temperature, hydration, thyroid gland examination, and breast examination for galactorrhoea was performed. A detailed systemic examination of the central nervous system, cardiovascular system, respiratory system, and abdomen was done. In sexually active women, speculum examinations and bimanual vaginal examinations were also done after taking consent. Routine investigations were done. Every participant in our study underwent pelvic ultrasound either by transabdominal or transvaginal route (depending on the preferences of the participant) to assess the uterus and ovaries.

Subjects were randomly assigned to one of the three treatment groups via a computer-generated code. Group 1 received berberine hydrochloride, $500 \mathrm{mg}$ twice daily. Group 2 received metformin hydrochloride, $500 \mathrm{mg}$ twice daily, and group 3 received myoinositol, $1000 \mathrm{mg}$ twice daily. No lifestyle changes were advised for any group, and subjects were encouraged to follow their normal diet and exercise routines. This was an open-label study. Both health professionals and patients were aware of the treatment being administered.

The outcomes studied were effects on clinical characteristics (weight, body mass index, waist circumference, waist-to-hip ratio), carbohydrate metabolic parameters (fasting plasma glucose, fasting insulin, fasting glucose/insulin ratio), hormonal parameters (total testosterone, free androgen index, sex hormone-binding globulin), and lipid profile (total cholesterol, triglycerides, low-density lipoprotein [LDL], very-low-density lipoprotein [VLDL], and high-density lipoprotein [HDL]) before and three months after treatment.

Data analyses were carried out using SPSS (software version: 13.0). Continuous variables were tested for normality assumptions using appropriate statistical tests. Descriptive statistics such as mean, standard deviation, and range values were calculated for normally distributed data, and mean values between the two groups were compared using Student's t independent test. The mean values between the three groups were compared using the analysis of variance (ANOVA) test. The level of significance in this study was taken as $p$ $<0.05$.

\section{Results}

Out of the total 136 patients recruited, 7 patients were excluded or lost to follow-up. Two patients in group 1 became pregnant, three patients in group 2 discontinued treatment due to gastrointestinal problems, and two patients in group 3 did not show up for follow-up examinations and investigations. A total of 129 patients were included in the analysis, 43 in each group.

In the current study, baseline characteristics were equally distributed within the groups. The mean age of the cases in group 1 was $27.57 \pm 4.81$ years, $27.67 \pm 5.06$ years in group 2 , and $26.57 \pm 4.81$ years in group $3(\mathrm{p}=$ $0.77)$. Patients of all age groups were equally present in all three groups. Rest parameters such as marital status, literacy, locality (rural/urban), occupation, and socioeconomic status were statistically insignificant between the groups ( $\mathrm{p}=0.99)$. Baseline biochemical parameters such as thyroid-stimulating hormone (TSH), prolactin, luteinizing hormone ( $\mathrm{LH}$ ), and follicle-stimulating hormone (FSH) were evenly distributed between the groups (Table 1). 


\section{Cureus}

\begin{tabular}{|c|c|c|c|c|c|}
\hline \multicolumn{2}{|l|}{ Parameters } & Group $1(n=43)$ & Group $2(n=43)$ & Group $3(n=43)$ & P-value \\
\hline Age & Mean \pm SD & $27.1 \pm 5.1$ & $27.67 \pm 5.06$ & $26.57 \pm 4.81$ & 0.77 \\
\hline \multirow{2}{*}{ Marital status } & Unmarried & $14(66.7 \%)$ & $13(61.9 \%)$ & $15(71.4 \%)$ & \multirow{2}{*}{0.99} \\
\hline & Married & $7(33.3 \%)$ & $8(39.1 \%)$ & $6(28.6 \%)$ & \\
\hline \multirow{2}{*}{ Locality } & Urban & $10(47.6 \%)$ & $11(52.3 \%)$ & 12 (52.1\%) & \multirow{2}{*}{0.99} \\
\hline & Rural & $11(52.3 \%)$ & 10 (47.6\%) & $9(42.9 \%)$ & \\
\hline \multirow{2}{*}{ Education } & Illiterate & $3(14.3 \%)$ & $2(9.5 \%)$ & $3(14.3 \%)$ & \multirow{2}{*}{0.99} \\
\hline & Literate & $18(85.7 \%)$ & 19 (90.5\%) & $18(85.7 \%)$ & \\
\hline \multirow{3}{*}{ Occupation } & Housewife & $5(23.8 \%)$ & $3(14.3 \%)$ & $6(28.6 \%)$ & \multirow{3}{*}{0.99} \\
\hline & Office worker & $6(28.5 \%)$ & 7 (33.3\%) & 7 (33.3\%) & \\
\hline & Student & $10(47.6 \%)$ & $11(52.4 \%)$ & $8(38.1 \%)$ & \\
\hline s.TSH ${ }^{*}$ & Mean \pm SD & $2.26 \pm 0.77$ & $2.43 \pm 0.72$ & $2.3 \pm 0.80$ & 00.554 \\
\hline s.Prolactin & Mean \pm SD & $25.6 \pm 11.00$ & $27.69 \pm 10.81$ & $27.85 \pm 12.17$ & 0.59 \\
\hline s.LH* & Mean \pm SD & $6.5 \pm 2.2$ & $7.6 \pm 3.6$ & $6.4 \pm 2.8$ & 0.10 \\
\hline s.FSH ${ }^{*}$ & Mean \pm SD & $5.4 \pm 2.02$ & $5.57 \pm 1.61$ & $5.57 \pm 1.76$ & 0.97 \\
\hline
\end{tabular}

\section{TABLE 1: Baseline characteristics}

*s.TSH: serum thyroid-stimulating hormone, *s.LH: serum luteinizing hormone, *s.FSH: serum follicle-stimulating hormone

\section{Clinical characteristics}

The decrease in mean weight, waist circumference (WC), waist-hip ratio (WHR), and body mass index (BMI) after 12 weeks of metformin, berberine, and myoinositol treatment was statistically significant in all groups individually ( $\mathrm{p}=0.0001$; Table 2 ). When comparing the clinical characteristics between the groups receiving metformin, berberine, and myoinositol after 12 weeks of therapy, the difference in weight and BMI was insignificant ( $p=0.0504$ and $p=0.7745$, respectively). However, WC decreased significantly between the three groups $(\mathrm{p}=0.0001)$. There was a greater reduction in WC in the Berberine group compared to the metformin group $(80.86 \pm 2.85$ vs $85.95 \pm 4.78)$ and the group receiving myoinositol $(80.86 \pm 2.85$ vs $88.24 \pm 3.77 ; \mathrm{p}>0.001)$. The WHR also showed a significant difference, i.e., a greater reduction in WHR was observed in the Berberine group compared to the metformin group $(0.82 \pm 0.03$ vs $0.87 \pm 0.06 ; \mathrm{p}=0.001)$ and myoinositol ( $0.82 \pm 0.03$ vs $0.89 \pm 0.04 ; \mathrm{p}=0.01)$ (Table 3). Metformin and myoinositol did not show any superiority over each other in reducing waist circumference and waist-hip ratio in the studied subjects. 


\section{Cureus}

\begin{tabular}{|c|c|c|c|c|c|c|c|c|c|}
\hline \multirow[b]{2}{*}{ Parameters } & \multicolumn{3}{|l|}{ Berberine } & \multicolumn{3}{|l|}{ Metformin } & \multicolumn{3}{|l|}{ Myoinositol } \\
\hline & Pre-treatment & Post-treatment & P-Value & Pre-treatment & Post-treatment & P-Value & Pre-treatment & Post-treatment & P-Value \\
\hline Weight & $62.07 \pm 5.24$ & $58.00 \pm 4.46$ & 0.0001 & $65.03 \pm 4.94$ & $60.81 \pm 4.05$ & 0.0001 & $63.06 \pm 4.74$ & $60.81 \pm 4.05$ & 0.0073 \\
\hline BMI & $24.69 \pm 2.99$ & $23.83 \pm 2.85$ & 0.0001 & $25.46 \pm 2.23$ & $23.87 \pm 1.94$ & 0.0001 & $24.35 \pm 2.24$ & $23.92 \pm 2.16$ & 0.0001 \\
\hline WC & $89.86 \pm 5.45$ & $85.95 \pm 4.78$ & 0.0001 & $88.9 \pm 4.61$ & $80.86 \pm 2.85$ & 0.0001 & $89.67 \pm 4.27$ & $88.24 \pm 3.77$ & 0.0001 \\
\hline WHR & $0.91 \pm 0.06$ & $0.87 \pm 0.06$ & 0.0001 & $0.89 \pm 0.06$ & $0.82 \pm 0.03$ & 0.0001 & $0.9 \pm 0.06$ & $0.89 \pm 0.04$ & 0.0002 \\
\hline FBS & $93.40 \pm 17.03$ & $81.31 \pm 15.55$ & 0.0001 & $90.54 \pm 15.04$ & $79.45 \pm 13.25$ & 0.0001 & $94.43 \pm 15.75$ & $79.46 \pm 13.35$ & 0.0001 \\
\hline S.FI & $21.94 \pm 2.89$ & $18.07 \pm 2.23$ & 0.0001 & $20.44 \pm 2.08$ & $15.93 \pm 1.7$ & 0.0001 & $21.74 \pm 2.02$ & $11.75 \pm 1.11$ & 0.0001 \\
\hline FPG/S.FI & $4.63 \pm 1.26$ & $6.21 \pm 2.13$ & 0.0001 & $4.71 \pm 1.03$ & $6.71 \pm 1.98$ & 0.0001 & $4.68 \pm 1.13$ & $6.91 \pm 1.68$ & 0.0001 \\
\hline s.TT & $1.9 \pm 6.9$ & $1.5 \pm 0.52$ & 0.0001 & $1.89 \pm 0.66$ & $1.53 \pm 0.51$ & 0.0001 & $1.93 \pm 0.7$ & $1.53 \pm 0.68$ & 0.0001 \\
\hline s.SHBG & $24.69 \pm 2.98$ & $54.22 \pm 6.5$ & 0.0001 & $25.46 \pm 2.24$ & $60.07 \pm 4.63$ & 0.0001 & $25.3 \pm 4.29$ & $54.18 \pm 3.83$ & 0.0001 \\
\hline FAl & $7.93 \pm 0.82$ & $3.02 \pm 0.68$ & 0.0001 & $7.7 \pm 0.86$ & $2.58 \pm 0.44$ & 0.0001 & $7.81 \pm 0.64$ & $3.03 \pm 0.61$ & 0.0001 \\
\hline s.TC & $5.79 \pm 0.07$ & $5.04 \pm 0.73$ & 0.0001 & $5.84 \pm 0.72$ & $4.52 \pm 0.6$ & 0.0001 & $5.75 \pm 0.65$ & $5.03 \pm 0.7$ & 0.0001 \\
\hline s.TG & $2.26 \pm 0.66$ & $2.12 \pm 0.64$ & 0.0001 & $2.49 \pm 0.6$ & $1.67 \pm 0.54$ & 0.0001 & $2.39 \pm 0.66$ & $2.19 \pm 0.62$ & 0.0001 \\
\hline s.LDL & $4.3 \pm 0.78$ & $3.58 \pm 0.6$ & 0.0001 & $4.23 \pm 0.86$ & $3.02 \pm 0.78$ & 0.0001 & $4.34 \pm 0.64$ & $2.51 \pm 0.54$ & 0.0001 \\
\hline s.HDL & $1.13 \pm 0.25$ & $1.19 \pm 0.24$ & 0.0001 & $1.09 \pm 0.27$ & $1.5 \pm 0.47$ & 0.0001 & $1.13 \pm 0.45$ & $1.19 \pm 0.46$ & 0.0001 \\
\hline
\end{tabular}

\section{TABLE 2: Comparison of different parameters in each group}

BMI: body mass index, WC: waist circumference, WHR: waist-hip ratio, FBS: fasting blood sugar, s.Fl: serum fasting insulin, s.TT: serum total testosterone, s.SHBG: serum sex hormone-binding globulin, FAI: free androgen index, s.TC: serum total cholesterol, s.TG: serum triglycerides, s.LDL: serum low-density lipoprotein, s.HDL: serum high-density lipoprotein 


\begin{tabular}{|c|c|c|c|c|c|c|c|c|}
\hline \multirow[b]{2}{*}{ Parameters } & \multicolumn{4}{|l|}{ Pre-treatment } & \multicolumn{4}{|c|}{ Post-treatment } \\
\hline & Berberine & Metformin & Myoinositol & P-value & Berberine & Metformin & Myoinositol & P-value \\
\hline Weight & $62.07 \pm 5.24$ & $65.03 \pm 4.94$ & $63.06 \pm 4.74$ & 0.0504 & $58.00 \pm 4.46$ & $60.81 \pm 4.05$ & $60.81 \pm 4.05$ & 0.0576 \\
\hline BMI & $24.69 \pm 2.99$ & $25.46 \pm 2.23$ & $24.35 \pm 2.24$ & 0.3006 & $23.83 \pm 2.85$ & $23.87 \pm 1.94$ & $23.92 \pm 2.16$ & 0.7745 \\
\hline WC & $89.86 \pm 5.45$ & $88.9 \pm 4.61$ & $89.67 \pm 4.27$ & 0.9616 & $85.95 \pm 4.78$ & $80.86 \pm 2.85$ & $88.24 \pm 3.77$ & 0.0001 \\
\hline WHR & $0.91 \pm 0.06$ & $0.89 \pm 0.06$ & $0.9 \pm 0.06$ & 0.8147 & $0.87 \pm 0.06$ & $0.82 \pm 0.03$ & $0.89 \pm 0.04$ & 0.0001 \\
\hline FBS & $93.40 \pm 17.03$ & $90.54 \pm 15.04$ & $94.43 \pm 15.75$ & 0.598 & $81.31 \pm 15.55$ & $79.45 \pm 13.25$ & $79.46 \pm 13.35$ & 0.9521 \\
\hline s.FI & $21.94 \pm 2.89$ & $20.44 \pm 2.08$ & $21.74 \pm 2.02$ & 0.0604 & $18.07 \pm 2.23$ & $15.93 \pm 1.7$ & $11.75 \pm 1.11$ & 0.0001 \\
\hline FBS/S.FI & $4.63 \pm 1.26$ & $4.71 \pm 1.03$ & $4.68 \pm 1.13$ & 0.2254 & $6.21 \pm 2.13$ & $6.71 \pm 1.98$ & $6.91 \pm 1.68$ & 0.0035 \\
\hline s.TT & $1.9 \pm 6.9$ & $1.89 \pm 0.66$ & $1.93 \pm 0.7$ & 0.998 & $1.5 \pm 0.52$ & $1.53 \pm 0.51$ & $1.53 \pm 0.68$ & 0.9953 \\
\hline s.SHBG & $24.69 \pm 2.98$ & $25.46 \pm 2.24$ & $25.3 \pm 4.29$ & 0.4653 & $54.22 \pm 6.5$ & $60.07 \pm 4.63$ & $54.18 \pm 3.83$ & 0.0003 \\
\hline FAl & $7.93 \pm 0.82$ & $7.7 \pm 0.86$ & $7.81 \pm 0.64$ & 0.6501 & $3.02 \pm 0.68$ & $2.58 \pm 0.44$ & $3.03 \pm 0.61$ & 0.0308 \\
\hline s.TC & $5.79 \pm 0.07$ & $5.84 \pm 0.72$ & $5.75 \pm 0.65$ & 0.9652 & $5.04 \pm 0.73$ & $4.52 \pm 0.6$ & $5.03 \pm 0.7$ & 0.0327 \\
\hline s.TG & $2.26 \pm 0.66$ & $2.49 \pm 0.6$ & $2.39 \pm 0.66$ & 0.5649 & $2.12 \pm 0.64$ & $1.67 \pm 0.54$ & $2.19 \pm 0.62$ & 0.0116 \\
\hline s.LDL & $4.3 \pm 0.78$ & $4.23 \pm 0.86$ & $4.34 \pm 0.64$ & 0.954 & $3.58 \pm 0.6$ & $3.02 \pm 0.78$ & $2.51 \pm 0.54$ & 0.0092 \\
\hline s.HDL & $1.13 \pm 0.25$ & $1.09 \pm 0.27$ & $1.13 \pm 0.45$ & 0.8093 & $1.19 \pm 0.24$ & $1.5 \pm 0.47$ & $1.19 \pm 0.46$ & 0.0437 \\
\hline
\end{tabular}

TABLE 3: Comparison of different parameters in between the groups (berberine vs metformin vs myoinositol)

BMI: body mass index, WC: waist circumference, WHR: waist-hip ratio, FBS: fasting blood sugar, s.FI: serum fasting insulin, s.TT: serum total testosterone, s.SHBG: serum sex hormone-binding globulin, FAl: free androgen index, s.TC: serum total cholesterol, s.TG: serum triglycerides, s.LDL: serum low-density lipoprotein, s.HDL: serum high-density lipoprotein

\section{Carbohydrate metabolic parameters}

All the groups had significant improvements in the carbohydrate metabolic parameters, fasting blood glucose (FBS), serum fasting insulin (FI), and FBS/S.FI after treatment ( $\mathrm{p}=0.0001$ ) (Table 2). Regarding FBG, after 3 months of treatment, the difference in mean value between the three groups before treatment and post-treatment remained statistically insignificant ( $\mathrm{p}=0.598$ and $\mathrm{p}=0.9521$, respectively) (Table 3). However, FI showed a significant difference between the three groups after treatment $(\mathrm{p}<0.0001)$. There was a greater reduction in serum fasting insulin in the group receiving myoinositol compared to the group receiving metformin or Berberine $(\mathrm{p}<0.0001)$. Regarding FBG/FI, a significant difference was observed between the three groups after treatment $(\mathrm{p}=0.0035)$. The group receiving myoinositol showed superiority over the group receiving metformin and berberine in increasing the FBG/FI ratio (Table 3).

\section{Hormonal parameters}

The decrease in TT, the increase in sex hormone-binding globulin (SHBG), and the reduction in the free androgen index (FAI) were significant after three months of treatment in all three groups ( $p=0.0001$; Table 1). After 12 weeks of therapy, no significant difference was found in mean values of s.total testosterone between the three groups $(\mathrm{p}=0.9953)$. The difference in mean s.SHBG between the three treatment groups was statistically significant ( $\mathrm{p}=0.0003$; Table 2 ). The increase in SHBG between the berberine receiving group was significantly higher than the metformin $(\mathrm{p}<0.01)$ and myoinositol receiving groups $(\mathrm{p}=0.001)$. A significant change in free FAI between the three groups was also observed $(\mathrm{p}=0.0308)$. The decrease in free androgen index in the group receiving berberine was significantly more than the group receiving myoinositol $(\mathrm{p}<0.05)$.

\section{Lipid profile}

All the groups showed a significant reduction in total cholesterol, serum triglyceride, serum LDL, and an increase in serum HDL ( $\mathrm{p}=0.0001$; Table 2 ). Between the groups, berberine showed a significant change over myoinositol and metformin in all four parameters (Table 3). Therefore, berberine supplementation improved the lipid profile parameter the most. 


\section{Discussion}

The present study compared the effects of three drugs on various parameters in reproductive-aged females suffering from PCOS. PCOS has diverse pathophysiology, and therefore different treatment options such as oral contraceptive pills (OCPs) alone or in combination with other agents have been used frequently [11].

Berberine has also been tested in some studies alone or in combination with OCPs $[4,12]$. In our study, we used berberine alone in group 1 . Treatment with berberine alone resulted in an improvement in clinical characteristics, carbohydrate metabolic parameters, and hormonal and lipid profiles (Table 2). Therefore, the present study demonstrated that berberine improves various parameters independently of OCPs in patients with PCOS. It has also been concluded by a meta-analysis that berberine in combination with OCPs is superior to OCPs alone [5]. The present study showed that berberine had an almost similar effect on various parameters as metformin and myoinositol, with the exception of some parameters like WC, WHR, SHBG, FAI, and lipid profile, where it showed significant improvement over other groups (Table 3). Wei et al. also showed significant improvement in WC, WHR, SHBG, and lipid profile with berberine when compared to metformin. However, they used OCPs and lifestyle modification as the primary interventions, along with the addition of berberine, metformin, and placebo randomly. In our study, subjects were restricted from using OCP or any targeted exercise program for the duration of the study (12 weeks). They were encouraged to follow their normal routine. Another study also confirmed our findings with an improvement in clinical, metabolic, and reproductive parameters with the use of berberine alone [4,12]. In a recent metanalysis of 12 RCTs on the effect of berberine on PCOS patients, it was reported that berberine improved reproductive hormones (TT, SHBG, LH, LH/FSH ratio), lowered fasting and post-prandial blood glucose, and improved lipid profile as compared to placebo/no treatment [13]. These findings are in congruence with the results obtained in our study. This improvement in reproductive hormones and metabolic parameters is explained by increased sensitivity to insulin and steroid hormone synthesis after berberine treatment, as demonstrated in other studies [14]. However, this study showed a significant improvement in WHR with no effect on BMI and WC with berberine compared to placebo/no treatment [13]. In our study, we observed a significant decrease in BMI, WC, and WHR before and after 12 weeks of berberine therapy. The difference in our findings could be explained by the difference in ethnicity of the study groups included in both studies. The same study also evaluated berberine and metformin comparatively and reported a significant change in hormone parameters (decrease in TT, increase in SHBG, and decrease in LH/FSH ratio), improvement in lipid profile, and change in body fat composition (decrease in WC and WHR with no change in BMI) with berberine supplementation as compared to metformin. However, there was no significant change in FBG, postprandial blood glucose, FI, and homeostatic model assessment of insulin resistance (HOMA-IR) between berberine and metformin therapy [13]. Our study also reciprocated the same findings. Improved insulin sensitivity, lipid profile, and redistribution of body fat induced by berberine offer a promising future prospect to prevent cardiovascular disease and metabolic syndrome in patients with PCOS [15].

Myoinositol, a precursor of the phosphatidylinositol second messenger pathway, has been explored as a therapeutic option for PCOS patients. Myoinositol has been reported to improve fertility outcomes due to its modulating effect on hyperandrogenism in PCOS patients [16]. Myoinositol has drawn attention recently and has been tried as a first-line drug for PCOS in many studies [17].

In our study, myoinositol led to improvement in all the parameters, that is, clinical, carbohydrate metabolic, hormonal, and lipid profile in group 3 after 12 weeks of therapy. Nehra et al. reported clear-cut improvements in weight, BMI, WC, and WHR with myoinositol therapy for 24 weeks. However, when comparing the effects with metformin, no significant differences were observed [18]. Another study also reported a significant change in BMI, WC, FBS, postprandial sugar, FI, TT, LH, and LH/FSH after myoinositol therapy. WHR did not decrease significantly in this study. Compared to metformin, they found no significant differences, except a significant decrease in HOMA-IR in the myoinositol group [6]. Similarly, metformin and myoinositol exhibited similar effects in the present study, with the exception of a significant decrease in FI and the FBG/FI ratio with myoinositol supplementation compared to metformin.

Chirania et al. also found a significant reduction in FI with the combined therapy of metformin and myoinositol. However, they did not detect any significant difference in FI when myoinositol was used alone. This difference in the findings could be due to a significant difference in the baseline FI between the groups in this study. In our study, there were no differences in baseline FI between the groups. A recent metanalysis also noticed significant changes in FBS, FI, and SHBG with myoinositol therapy. This study reported homogeneous findings with both metformin and myoinositol therapy, except for a significant reduction in androgens with myoinositol therapy [17].

Metformin has been used in PCOS patients with a good response for days beyond the recall. Our study has shown that metformin therapy improves all parameters in PCOS patients. However, PCOS is a continuum condition in the life of a woman. Long-term therapy with metformin is unacceptable for some due to its adverse gastrointestinal side effects [16]. In that case, as shown in our study, alternative therapy could be tried with similar benefits in women with PCOS.

The main advantage of this study is the prospective random allocation of patients to all the groups. Second, all the groups were similar at the baseline, achieving adequate matching. Third, this study compared three 
different drugs in PCOS patients. Fourth, strict inclusion and exclusion criteria were applied in recruiting participants. The disadvantages of this study are that blinding was not done (this was an open-label study), so biases cannot be excluded completely. However, we did not include subjective symptoms such as irregular menses, hair growth, acne, etc., in the evaluated results, which could introduce potential biases into the study. Second, the outcomes like pregnancy rate and live birth rate were not studied in the study.

\section{Conclusions}

Metformin has been used and will continue to be used in PCOS patients. It is the oldest and safest insulin sensitizer for women suffering from PCOS. However, long-term compliance with metformin could be troublesome for some PCOS patients. So, the present study explored two alternative drugs, berberine, and myoinositol. Berberine was associated with an improvement in various measures of insulin resistance that was well comparable to that of metformin. The notable risk factors for metabolic syndrome and cardiovascular disease are increased WC, WHR, and a deranged lipid profile. Berberine improved all these parameters, and the data suggest that berberine may have a greater potential to reduce cardiovascular disease risk than metformin in patients with PCOS. In this study, myoinositol was shown to significantly improve insulin sensitivity compared to metformin. Myoinositol could be the game-changer in this regard, as PCOS patients are at high risk of developing diabetes in later life. Myoinositol shares clinical and hormonal benefits similar to metformin. It may be considered as a first-line option in PCOS patients with insulin resistance without prediabetes or diabetes.

\section{Additional Information \\ Disclosures}

Human subjects: Consent was obtained or waived by all participants in this study. Institutional ethics Committee MLNMC issued approval IEC/MLNMC2014/No.02 (MLNMC-Motilal Nehru Medical College). After consideration, the committee has decided to approve the study under the referenced protocol. Animal subjects: All authors have confirmed that this study did not involve animal subjects or tissue. Conflicts of interest: In compliance with the ICMJE uniform disclosure form, all authors declare the following: Payment/services info: All authors have declared that no financial support was received from any organization for the submitted work. Financial relationships: All authors have declared that they have no financial relationships at present or within the previous three years with any organizations that might have an interest in the submitted work. Other relationships: All authors have declared that there are no other relationships or activities that could appear to have influenced the submitted work.

\section{Acknowledgements}

We sincerely appreciate the efforts of Late Professor Meena Dayal in helping in participants recruitment.

\section{References}

1. Ganie MA, Kalra S: Polycystic ovary syndrome - a metabolic malady, the mother of all lifestyle disorders in women - can Indian health budget tackle it in future?. Indian J Endocrinol Metab. 2011, 15:239-41. $10.4103 / 2230-8210.85571$

2. Gurbuz T, Alanya Tosun S, Cebi A, Gokmen O, Usta M: Investigating fetuin-a and paraoxonase-1 activity as markers in polycystic ovary syndrome based on body mass index: a prospective case-control study. Cureus. 2021, 13:e18553. 10.7759/cureus.18553

3. Viollet B, Guigas B, Sanz Garcia N, Leclerc J, Foretz M, Andreelli F: Cellular and molecular mechanisms of metformin: an overview. Clin Sci (Lond). 2012, 122:253-70. 10.1042/CS20110386

4. Wei W, Zhao H, Wang A, et al.: A clinical study on the short-term effect of berberine in comparison to metformin on the metabolic characteristics of women with polycystic ovary syndrome. Eur J Endocrinol. 2012, 166:99-105. 10.1530/EJE-11-0616

5. Li MF, Zhou XM, Li XL: The effect of berberine on polycystic ovary syndrome patients with insulin resistance (PCOS-IR): a meta-analysis and systematic review. Evid Based Complement Alternat Med. 2018, 2018:2532935. 10.1155/2018/2532935

6. Angik R, Jajoo SS, Hariharan C, Chimote A: A comparative study of metabolic and hormonal effects of myoinositol vs. metformin in women with polycystic ovary syndrome: a randomised controlled trial. Int J Reprod Contracept Obstet Gynecol. 2015, 4:189-194.

7. Genazzani AD, Lanzoni C, Ricchieri F, Jasonni VM: Myo-inositol administration positively affects hyperinsulinemia and hormonal parameters in overweight patients with polycystic ovary syndrome. Gynecol Endocrinol. 2008, 24:139-44. 10.1080/09513590801893232

8. Dumitrescu R, Mehedintu C, Briceag I, Purcărea V, Hudita D: Metformin-clinical pharmacology in PCOs. J Med Life. 2015, 8:187-92.

9. Christofides EA: Practical insights into improving adherence to metformin therapy in patients with type 2 diabetes. Clin Diabetes. 2019, 37:234-41. 10.2337/cd18-0063

10. Wang R, Mol BW: The Rotterdam criteria for polycystic ovary syndrome: evidence-based criteria? . Hum Reprod. 2017, 32:261-4. 10.1093/humrep/dew287

11. Badawy A, Elnashar A: Treatment options for polycystic ovary syndrome. Int J Womens Health. 2011, 3:2535. 10.2147/IJWH.S11304

12. Orio F, Muscogiuri G, Palomba S, et al.: Berberine improves reproductive features in obese Caucasian women with polycystic ovary syndrome independently of changes of insulin sensitivity. e-SPEN J. 2013, 


\section{Cureus}

8:e200-204. 10.1016/j.clnme.2013.07.002

13. Xie L, Zhang D, Ma H, et al.: The effect of berberine on reproduction and metabolism in women with polycystic ovary syndrome: a systematic review and meta-analysis of randomized control trials. Evid Based Complement Alternat Med. 2019, 2019:7918631. 10.1155/2019/7918631

14. Wang Z, Nie K, Su H, Tang Y, Wang H, Xu X, Dong H: Berberine improves ovulation and endometrial receptivity in polycystic ovary syndrome. Phytomedicine. 2021, 91:153654. 10.1016/j.phymed.2021.153654

15. Feng X, Sureda A, Jafari S, et al.: Berberine in cardiovascular and metabolic diseases: from mechanisms to therapeutics. Theranostics. 2019, 9:1923-51. 10.7150/thno.30787

16. Azizi Kutenaei M, Hosseini Teshnizi S, Ghaemmaghami P, Eini F, Roozbeh N: The effects of myo-inositol vs. metformin on the ovarian function in the polycystic ovary syndrome: a systematic review and metaanalysis. Eur Rev Med Pharmacol Sci. 2021, 25:3105-15. 10.26355/eurrev_202104_25565

17. Chirania K, Misra S, Behera S: A randomised clinical trial comparing myoinositol and metformin in PCOS . Int J Reprod Contracept Obstet Gynecol. 2017, 6:1814-1820. 10.18203/2320-1770.ijrcog20171563

18. Nehra J, Kaushal J, Singhal SR, Ghalaut VS.: Comparison of myo-inositol versus metformin on anthropometric parameters in polycystic ovarian syndrome in women. Int J Pharm Pharm Sci. 2017, 9:144148 